$11-1-2021$

Features on cardiac magnetic resonance imaging and accuracy of echocardiographic findings for diagnosing constrictive pericarditis

Salik Ur Rehman Iqbal

Fateh Ali Tipoo Sultan

Follow this and additional works at: https://ecommons.aku.edu/pakistan_fhs_mc_med_cardiol

Part of the Cardiology Commons, and the Cardiovascular Diseases Commons 


\section{Features on cardiac magnetic resonance imaging and accuracy of echocardiographic findings for diagnosing constrictive pericarditis}

Salik ur Rehman Iqbal1, Fateh Ali Tipoo Sultan²

\begin{abstract}
Constrictive pericarditis is a rare disease with a difficult diagnosis. Cardiac magnetic resonance (CMR) imaging data of Aga Khan University Hospital from January 2011 to March 2020 was retrospectively reviewed and patients with the diagnosis of constrictive pericarditis were included.

A total of 22 patients were included with the mean age of $46 \pm 16$ years and majority $17(77 \%)$ were male. The most common findings on transthoracic echo were significant respiratory variation in mitral and tricuspid inflow velocities in 20(91\%), and septal annular $e^{\prime}>9$ in 10 (86\%). The most common finding on CMR was respiratory septal shift in $22(100 \%)$, followed by septal bounce in $21(95 \%)$ and thickened pericardium in $18(82 \%)$. Nearly two-third of the patients, $15(70 \%)$ were considered for pericardiectomy but it was deferred in 5 patients due to high surgical risk. Ten patients underwent pericardiectomy, with no mortality on a mean follow up of $4 \pm 2$ years.
\end{abstract}

Keywords: Constrictive pericarditis (CP), Cardiac MRI (CMR), Transthoracic echocardiogram (TTE).

\section{DOI: https://doi.org/10.47391/JPMA.01346}

\section{Introduction}

Constrictive pericarditis (CP) is a rare disease with a difficult diagnosis. It is a form of diastolic heart failure, caused by a rigid and inelastic pericardium that prevents adequate ventricular filling. In developed countries, prior cardiac surgery, irradiation therapy, and idiopathic pericarditis are the most frequent causes of constrictive pericarditis. However, in developing countries, tuberculosis still constitutes a major cause of CP. ${ }^{1}$ CMR has far greater accuracy and reliability in diagnosing $\mathrm{CP}$ compared to transthoracic echo (TTE) and may also provide details on haemodynamics. Combination of pericardial thickness and septal shift has a high sensitivity and specificity.

No data is available from Pakistan on CMR features of constrictive pericarditis. As tuberculosis is the usual cause of constrictive pericarditis in developing countries like 1Department of Cardiology, Aga Khan University Hospital, Karachi, Pakistan; 2Department of Medicine and Cardiology, Aga Khan University Hospital, Karachi, Pakistan.

Correspondence: Salik ur Rehman Iqbal. e-mail: rehman.salik@yahoo.com
Pakistan, it would be important to see the echo findings and CMR features in these patients. We aimed to do the study to see the CMR features and echocardiographic findings in constrictive pericarditis, in the Pakistani population presenting to a tertiary care hospital.

\section{Case Series}

This study was approved by hospital ethical review committee (ERC No. 2020-4775-10220). Cardiac magnetic resonance imaging data of Aga Khan University Hospital, from January 2011 to March 2020 was retrospectively reviewed and patients with the diagnosis of constrictive pericarditis were included. These patients had clinical and echocardiographic features of constrictive pericarditis and were referred for CMR for confirmation of the diagnosis. On CMR, respire-phasic septal shift was considered diagnostic, in the presence of supportive features like dilated atria, dilated IVC, septal bounce and pericardial thickening. ${ }^{2}$ All the patients with a medial mitral $\mathrm{e}^{\prime}<6 \mathrm{~cm} / \mathrm{s}, \mathrm{E} / \mathrm{A}>2$, respiratory variations of $<10 \%$ on mitral inflow and absence of annulus reversus on echo were considered to have restrictive cardiomyopathy and were excluded from the study. Similarly patients with features of restrictive cardiomyopathy on CMR, like characteristic patterns of myocardial delayed enhancement with absence of pericardial thickening and absence of septal shift were also excluded from the study.

A pre designed data collection form was filled for each patient after reviewing the medical records. Data was analyzed using SPSS version 22. CMR was performed on 1.5-Tesla Siemens Avanto.

A total of 22 patients were included with the mean age of $46 \pm 16$ years and majority $17(77 \%)$ were male. Recent or past history of tuberculosis was present in $11(50 \%)$, while $6(27 \%)$ had clinical suspicion of tuberculosis (Table).

The most common findings on TTE supporting the diagnosis of $\mathrm{CP}$ were significant respiratory variation in mitral and tricuspid inflow velocities in 20(91\%) and septal annular $\mathrm{e}^{\prime}>9$ in 19(86\%).

On TTE, respiratory septal shift was present in 9(41\%), septal bounce in $14(64 \%)$, biatrial enlargement in 15(68\%), dilated IVC in 15(68\%) and thickened pericardium in 
Table: Baseline data, imaging features on Transthoracic Echocardiogram and Cardiac Magnetic Resonance Imaging and outcomes of patients with constrictive pericarditis.

\begin{tabular}{lll}
\hline & & $\mathbf{n}(\%)$ \\
\hline Mean Age (years) & $46 \pm 16$ & \\
Gender & Males & $17(77)$ \\
& Females & $5(23)$ \\
Comorbid & Recent or past history of tuberculosis & $11(50)$ \\
& Suspected Tuberculosis (on empirical ATT*) & $6(27)$ \\
& History of Cardiac surgery & $2(09)$ \\
& Others (Brucellosis) & $1(04)$ \\
Common Non-invasive & Transthoracic Echocardiogram & - \\
parameters & n (\%) & Cardiac MRI \\
Respiratory septal shift & 9 (41) & $\mathbf{n}(\%)$ \\
Septal bounce & 14 (64) & $22(100)$ \\
Dilated IVC & $15(68)$ & $21(95)$ \\
Thickened pericardium & 15 (68) & $19(86)$ \\
Other Non-invasive & Respiratory variation: 20 (91) & $18(82)$ \\
parameters & Septal e' $>$ 9: 19 (86) & LGE: 17 (77) \\
& Annulus reversus: 14 (64) & \\
Follow-up & Hepatic vein flow reversal: 12 (54) & \\
& Duration (years) & $4.0 \pm 2.0$ \\
& Surgery advised & $15(68)$ \\
& Patients who underwent surgery & $10(45)$ \\
& Mortality (before surgery) & $1(4)$ \\
\hline & Mortality (after surgery) & - \\
\hline
\end{tabular}

15 (68\%). Expiratory hepatic vein flow reversal was seen in $12(54 \%)$.

The most common finding on CMR was respiratory septal shift in all 22(100\%), followed by septal bounce in $21(95 \%)$, dilated IVC in 19 (86\%), and thickened pericardium in $18(82 \%)$. Pericardial LGE on CMR was seen in $17(77 \%)$ (Figure).
Mean follow up of $4 \pm 2.0$ years was available. More than half, $12(54 \%)$ had signs and symptoms of right heart failure. Nearly two-third of the patients, 15(68\%) were considered for pericardiectomy, but it was deferred in 5 patients due to high surgical risk. Ten patients underwent pericardiectomy with non-specific inflammation as the most common histological finding, and no mortality was encountered on follow up.

\section{Discussion}

This is a small case series of a rare disease. It is the first study from Pakistan which describes the echocardiographic and CMR features of constrictive pericarditis. Its importance further increases, as it is from a region where tuberculosis is considered the most common cause of constrictive pericarditis. Although a CT scan is widely available and valuable in detecting pericardial calcification and thickening, a third of the patients with constrictive pericarditis do not have a thickened pericardium. Furthermore, as constrictive pericarditis is a haemodynamic process, the requirement for breath-holding during imaging limits assessment of ventricular interdependence reducing its sensitivity. Also, CT scans subject the patients to radiation exposure. These limitations of $\mathrm{CT}$ scans are offset by the advantage of CMR that has high sensitivity and specificity for respire-phasic septal shift and can even diagnose constrictive pericarditis in the absence of pericardial thickening. In addition, CMR can also exclude other causes of similar presentations like cases of restrictive cardiomyopathy.

A majority of the patients in this study either had a history of tuberculosis (TB) or clinical suspicion of tuberculosis, which is different from developed countries where idiopathic causes, cardiac surgery and mediastinal

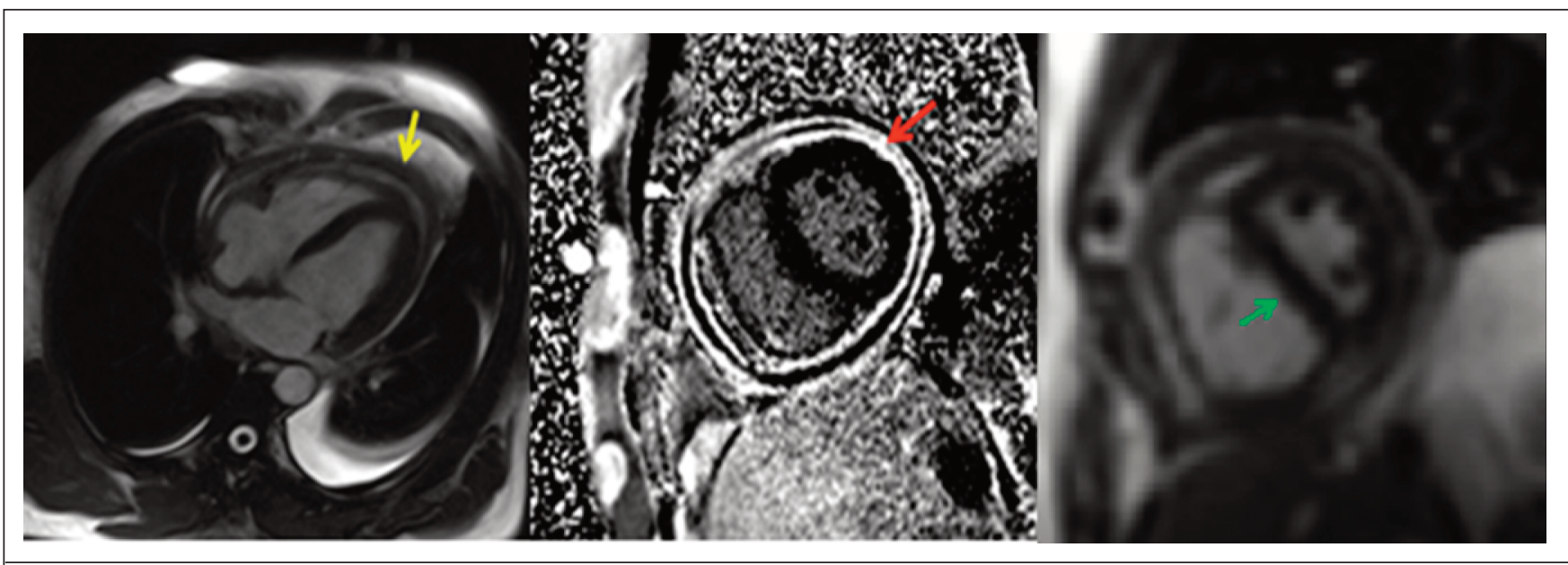

Figure: 1-A: SSFP image - Still frame showing thickened pericardium (yellow arrow) and left sided pleural effusion. 1-B: LGE image - short axis view showing hyper-enhanced both layers of pericardium (red arrow) and minimal pericardial effusion. 1-C: Real time CMR image without breath hold - Still frame showing septal shift (green arrow) towards left ventricle during inspiration. 
irradiation are common aetiologies of constrictive pericarditis. In this study, only two of the patients had a history of cardiac surgery and no patient had a history of radiation exposure.

Diagnosis of constrictive pericarditis and its differentiation from restrictive cardiomyopathy is important but usually difficult. TTE is the initial and sometimes the only affordable test for the diagnosis of this condition. As per Hancock, septal bounce, ventricular septal shift with respiration and bi-atrial enlargement are the three basic signs on TTE in constrictive pericarditis. 3 In our study, septal bounce was present in $64 \%$ and septal shift in $41 \%$ while bi-atrial enlargement was seen in $68 \%$, on TTE.

Significant respiratory variation in mitral and tricuspid inflow velocities represents ventricular interdependence and is an important pathophysiologic feature in $\mathrm{CP}$, which was present in $91 \%$ of the patients in this study. This feature is not present in restrictive cardiomyopathy and a number of constrictive pericarditis patients also do not demonstrate this sign.

According to a study by Welch, ${ }^{4}$ the combination of respiratory septal shift with either a medial $e^{\prime}$ velocity $>9$ $\mathrm{cm} / \mathrm{sec}$ or a hepatic vein expiratory diastolic reversal ratio of $>0.79$ ensures a diagnostic sensitivity of $87 \%$ and a specificity of $91 \%$. If all three factors coexist, the specificity rises to $97 \%$ compromising the sensitivity to $64 \%$.

In this study, expiratory hepatic vein flow reversal was seen in more than half of the patients and septal e $>9 \mathrm{~cm} / \mathrm{s}$ in $86 \%$ patients.

CMR is considered a very reliable imaging modality for diagnosing constrictive pericarditis. However, its use is limited due to less availability and more cost. In two CMR studies on CP, septal bounce had a sensitivity of $81 \%$ to $96 \%$ and a specificity of $100 \%$ for the diagnosis of CP whereas on TTE, it has a sensitivity of $62 \%$ and specificity of $93 \%$ for CP diagnosis.5,6 In our study, septal bounce was seen in $95 \%$ on CMR and $64 \%$ on TTE.

On CMR, combination of pericardial thickness and respiratory septal shift achieve a sensitivity of $100 \%$ and specificity of $90 \%$ in diagnosing CP. ${ }^{7}$ In our study, respiratory septal shift was seen in $100 \%$ of patients on CMR, whereas for the same patients it was picked up in $41 \%$ on TTE. In comparison to CT and CMR, TTE is less reliable in detecting thickened pericardium. We also observed that increased pericardial thickness was detected in $82 \%$ by CMR compared to $68 \%$ by TTE.

It is important to note that $12-18 \%$ of patients undergoing pericardiectomy for constrictive pericarditis, show normal pericardial thickness on histology. 8 Normal pericardial thickness in $18 \%$ of patients on CMR in our study, correlates with the percentage of histologically proven normal pericardial thickness in the above study. Pericardiectomy is the definitive treatment especially in advanced cases, and it is recommended for most patients. ${ }^{9}$ However, a trial of conservative treatment can also be considered in sub-acute form of constrictive pericarditis. In our study, nearly twothird of the patients were considered for pericardiectomy, but the procedure was deferred in 5 patients due to high surgical risk.

According to a study, in chronic constrictive pericarditis patients' survival is higher following pericardiectomy as compared to that of individuals without surgery. 10 However, choosing the right time for pericardiectomy is important. Only ten of the patients underwent pericardiectomy in our study with no mortality on follow-up.

Despite clinical diagnosis of tuberculous constrictive pericarditis and treatment with anti-tuberculous therapy, histopathology revealed non-specific inflammation in the majority of patients who underwent pericardiectomy. The granulomas may be missed on the tissue samples used for histopathology due to patchy involvement of the pericardium. The other explanation to this discrepancy is the fact that tuberculous constrictive pericarditis occurs at a late, burnt out stage of TB when it is difficult to demonstrate typical granulomas in the pericardium.

\section{Conclusion}

$\mathrm{CP}$ is better diagnosed by CMR than TTE. On TTE, significant respiratory variation in mitral and tricuspid inflow velocities and septal annular $e^{\prime}>9 \mathrm{~cm} / \mathrm{s}$ are commonly seen. Respiratory septal shift, septal bounce and thickened pericardium are common findings on CMR, followed by LGE. Patients treated with pericardiectomy show a good outcome. The most probable etiology of constrictive pericarditis in this region, is tuberculosis although it is difficult to prove TB on histopathology due to either burnt out stage of the disease or patchy involvement of the pericardium.

\section{Limitations}

This study was limited by the capability of Cardiac MRI to detect pericardial calcification and that this modality is not widely available. However, pericardial calcification is only found in constrictive pericarditis due to tuberculosis and this is also associated with pericardial thickening and other haemodynamic features of constrictive pericarditis which are easily picked up on CMR.

Disclaimer: This study is neither presented before nor 
published in any journal.

\section{Conflict of interest: None.}

\section{Funding Disclosure: None.}

\section{References}

1. Oh KY, Shimizu M, Edwards WE, Tazelaar HD, Danielson GK. Surgical pathology of the parietal pericardium: a study of 344 cases (19931999). Cardiovasc Pathol. 2001; 10:157-68.

2. Klein AL, Abbara S, Agler DA, Appleton CP, Asher CR, Hoit B, et al. American Society of Echocardiography clinical recommendations for multimodality cardiovascular imaging of patients with pericardial disease: endorsed by the Society for Cardiovascular Magnetic Resonance and Society of Cardiovascular Computed Tomography. J Am Soc Echocardiogr. 2013; 26:965-1012.e15.

3. Hancock EW. Differential diagnosis of restrictive cardiomyopathy and constrictive pericarditis. Heart. 2001; 86:343-9.

4. Welch TD, Ling LH, Espinosa RE, Anavekar NS, Wiste HJ, Lahr BD, et al. Echocardiographic diagnosis of constrictive pericarditis: Mayo Clinic criteria. Circ Cardiovasc Imaging. 2014; 7:526-34.
5. Walker CM, Chung JH, Reddy GP. Septal bounce. J Thorac Imaging 2012; 27: w1.

6. Dal-Bianco JP, Sengupta PP, Mookadam F, Chandrasekaran K, Tajik AJ, Khandheria BK. Role of echocardiography in the diagnosis of constrictive pericarditis. J Am Soc Echocardiogr. 2009; 22:24-33.

7. Bolen MA, Rajiah P, Kusunose K, Collier P, Klein A, Popović ZB, et al. Cardiac MR imaging in constrictive pericarditis: multiparametric assessment in patients with surgically proven constriction. Int J Cardiovasc Imaging. 2015; 31:859-66.

8. Talreja DR, Edwards WD, Danielson GK, Schaff HV, Tajik AJ, Tazelaar $H D$, et al. Constrictive pericarditis in 26 patients with histologically normal pericardial thickness. Circulation. 2003; 108:1852-7.

9. Maisch B, Seferović PM, Ristić AD, Erbel R, Rienmüller R, Adler Y, et al. Guidelines on the diagnosis and management of pericardial diseases. Executive summary: The task force on the diagnosis and management of pericardial diseases of the European Society of Cardiology. Eur Heart J. 2004; 25:587-610.

10. Yang HS, Song JK, Song JM, Kang DH, Lee CW, Nam GB, et al. Clinical characteristics of constrictive pericarditis diagnosed by echoDoppler technique in Korea. J Korean Med Sci 2001; 16:558-66. 